\title{
Interaction between the selectivity filter and the fast inactivation machinery in the voltage-gated $\mathrm{Na}^{+}$channel René Cervenka, Touran Zarrabi, Xaver Koenig, Eva-Maria Zebedin, Karlheinz Hilber and Hannes Todt*
}

\author{
Address: Centre for Biomolecular Medicine and Pharmacology, Medical University of Vienna, Austria \\ Email: Hannes Todt* - hannes.todt@meduniwien.ac.at \\ * Corresponding author
}

\author{
from I 3th Scientific Symposium of the Austrian Pharmacological Society (APHAR). Joint Meeting with the Austrian Society of Toxicology (ASTOX) and the \\ Hungarian Society for Experimental and Clinical Pharmacology (MFT) \\ Vienna, Austria. 22-24 November 2007 \\ Published: 14 November 2007 \\ BMC Pharmacology 2007, 7(Suppl 2):A15 doi:10.1 I86/I47|-22I0-7-S2-A I5
}

This abstract is available from: http://www.biomedcentral.com/I47I-22/0/7/S2/AI5

(C) 2007 Cervenka et al; licensee BioMed Central Ltd.

In the voltage-gated $\mathrm{Na}^{+}$channel the central pore is believed to be lined by the S6 segments of all four domains. Conformational changes of these S6 segments are thought to give rise to channel opening, closing and fast inactivation (FI). Whereas FI most likely occurs by an occlusion of the intracellular part of the pore, the selectivity filter of the channel is located in the extracellular vestibule. We sought to investigate possible interactions between the selectivity filter and the intracellular part of the domain IV S6 segment which is known be involved in FI. To this end, a critical residue within the selectivity filter of the $\mathrm{rNa}_{\mathrm{V}} 1.4$ channel, K1237 was replaced by the negatively charged glutamate (K1237E). This mutation was combined with serial cysteine replacements of amino acids in the S6 segment of domain IV. In K1237E the midpoint of FI (V05) was shifted to the hyperpolarized direction relative to wild-type ( $-60 \pm 13$ vs. $-47 \pm 11 \mathrm{mV}, \mathrm{n}=6$, $\mathrm{p}<0.01)$. Mutations of 16 residues in the domain IV-S6 produced inconsistent changes of V05. However, when these mutations were combined with K1237E, V05 was shifted to more negative values in all but one double mutants (mean shift $-13 \mathrm{mV}$ ), irrespective of the direction and the amount of shift produced by the single $S 6$ mutation. Conclusion: The selectivity filter of the voltage-gated $\mathrm{Na}^{+}$channel is coupled to the machinery of FI.

\section{Acknowledgements}

Support: Austrian Science Fund PI7509-BII. 